ARTICLE

DOI: $10.1038 / \mathrm{s} 41467-017-01988-1$

\title{
Evidence for a topological excitonic insulator in InAs/GaSb bilayers
}

Lingjie Du¹, Xinwei Li², Wenkai Lou (10 ${ }^{3}$, Gerard Sullivan ${ }^{4}$, Kai Chang ${ }^{3}$, Junichiro Kono ${ }^{1,2,5}$ \& Rui-Rui Du ${ }^{1,6}$

Electron-hole pairing can occur in a dilute semimetal, transforming the system into an excitonic insulator state in which a gap spontaneously appears at the Fermi surface, analogous to a Bardeen-Cooper-Schrieffer (BCS) superconductor. Here, we report optical spectroscopic and electronic transport evidence for the formation of an excitonic insulator gap in an inverted InAs/GaSb quantum-well system at low temperatures and low electron-hole densities. Terahertz transmission spectra exhibit two absorption lines that are quantitatively consistent with predictions from the pair-breaking excitation dispersion calculated based on the BCS gap equation. Low-temperature electronic transport measurements reveal a gap of $\sim 2 \mathrm{meV}$ (or $\sim 25 \mathrm{~K}$ ) with a critical temperature of $\sim 10 \mathrm{~K}$ in the bulk, together with quantized edge conductance, suggesting the occurrence of a topological excitonic insulator phase.

\footnotetext{
${ }^{1}$ Department of Physics and Astronomy, Rice University, Houston, TX 77005, USA. ${ }^{2}$ Department of Electrical and Computer Engineering, Rice University, Houston, TX 77005, USA. ${ }^{3}$ SKLSM, Institute of Semiconductors, Chinese Academy of Sciences, Beijing 100083, China. ${ }^{4}$ Teledyne Scientific and Imaging, Thousand Oaks, CA 91630, USA. 5 Department of Materials Science and NanoEngineerng, Rice University, Houston, TX 77005, USA. ${ }^{6}$ ICQM, Peking University, Beijing 10083, China. Correspondence and requests for materials should be addressed to K.C. (email: kchang@semi.ac.cn) or to J.K. (email: Kono@rice.edu) or to R.-R.D. (email: rrd@rice.edu)
} 
$\mathrm{t}$ was predicted several decades ago ${ }^{1,2}$ that Coulomb interactions in an electron-hole $(\mathrm{e}-\mathrm{h})$ co-existing system can make the normal semimetallic state unstable against the spontaneous appearance of excitons, or bound $\mathrm{e}-\mathrm{h}$ pairs, inducing a phase transition into an insulator, called the excitonic insulator (EI) or Bardeen-Cooper-Schrieffer (BCS)-like excitonic condensation. The EI phase emerges below a density-dependent critical temperature (Fig. 1a), where a gap opens at the energy of the original Fermi surface of the semimetal, in a manner analogous to the BCS gap in a superconductor ${ }^{3-9}$. In the density regime where the EI phase is expected to occur from semimetal (Fig. 1b), the spatial extent of the exciton wavefunction is larger than the average inter-exciton distance, i.e., electrons and holes are only weakly bound (Fig. 1c), similar to Cooper pairs. Conceptually, this density regime is distinct from the dilute limit where a quantum-degenerate gas of tightly bound e-h pairs (Fig. 1d) is transformed into a Bose-Einstein condensate (BEC) with macroscopic coherence (Fig. 1e). The possibility of excitonic ground state formation is enhanced in two-dimensional (2D) systems due to reduced screening. There have been extensive experiments on the possible appearance of nonequilibrium BEC in photoexcited $2 \mathrm{D}$ e-h systems probed by photoluminescence ${ }^{10,11}$, and equilibrium BEC state in quantum Hall electron-electron double-layers probed by counter-flow measurements ${ }^{12}$ and light scattering ${ }^{13}$. Note that these two types of BEC states have distinctions, besides the way to prepare BEC state, the former state is a short lived metastable superfluid supporting interference and vortices, whereas the latter is a ground state of the system. However, the EI side of the phase diagram has not been experimentally explored in any $2 \mathrm{D}$ system.

The originally proposed EI was based on a low density, equilibrium $\mathrm{e}-\mathrm{h}$ gas that exists in certain semimetals, and the possibility of the EI phase was systematically studied in the vicinity of a pressure-tuned semimetal-semiconductor transition ${ }^{5}$. Highly controllable 2D semiconductor materials emerged in ensuing years. In particular, InAs/GaSb quantum wells (QWs) exhibit unique inverted band structure with finite overlap of the conduction and valance bands, allowing the coexistence of spatially separated electrons and holes without photoexcitation, which offers a natural setting for equilibrium excitons and consequent formation of condensates including the EI phase $e^{9,14,15}$. Electrons are located in the InAs QW and holes are located in the GaSb QW, and thus, they are spatially separated in real space. The average separation can be defined as one half of the thickness of the double-QW structure. In this system, signatures of magneto-excitons were previously reported ${ }^{16}$. Recently, the quantum spin Hall (QSH) effect was explored in such QWs ${ }^{17-20}$. Robust quantized edge transport was observed ${ }^{19}$, persisting even at strong magnetic fields and high temperatures; it was theoretically suggested ${ }^{20}$ that the formation of a topological $\mathrm{EI}^{21}$ state may account for these unexpected properties. A topological EI in $2 \mathrm{D}$ has helical edge states propagating on the perimeters of a bulk EI state.

Here, we report optical spectroscopic and electronic transport evidence for the appearance of a BCS-like excitonic insulator gap in gated InAs/GaSb QW devices (Fig. 1f) at low temperatures with a low intrinsic e-h pair density, $n_{0} \sim p_{0} \sim 5.5 \times 10^{10} \mathrm{~cm}^{-2}$ (we use $n$ and $p$ to denote, respectively, the band electron and hole densities, and in particular, $n_{0}$ and $p_{0}$ denote the electron and hole densities at the charge-neutrality point). Our temperature- and magnetic field-dependent terahertz $(\mathrm{THz})$ transmission spectroscopy data can be quantitatively explained through our calculated pair-breaking excitation dispersion, $E(\mathrm{k})$, of the presumed EI state formed in the system. Complementarily, our low-temperature a
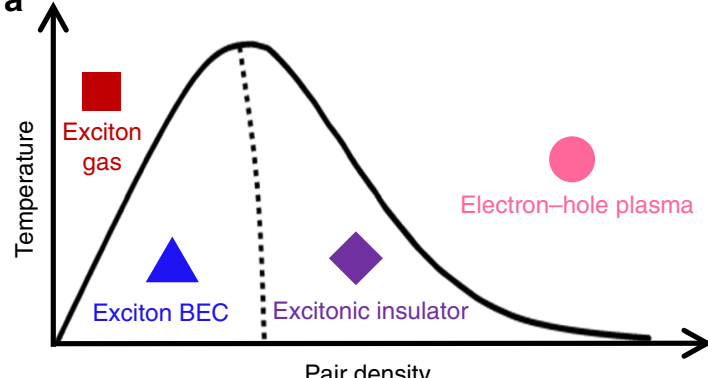

b

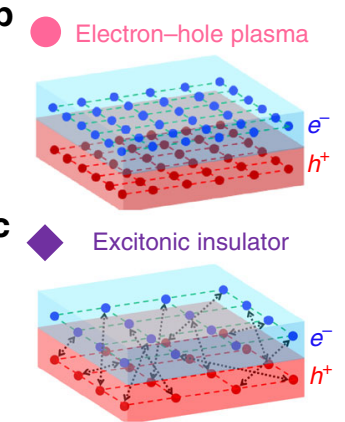

d

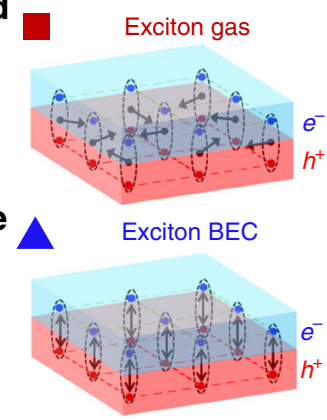

$f$

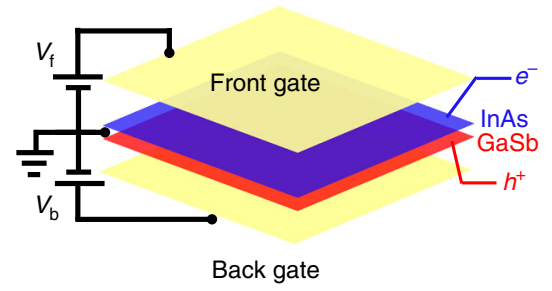

9

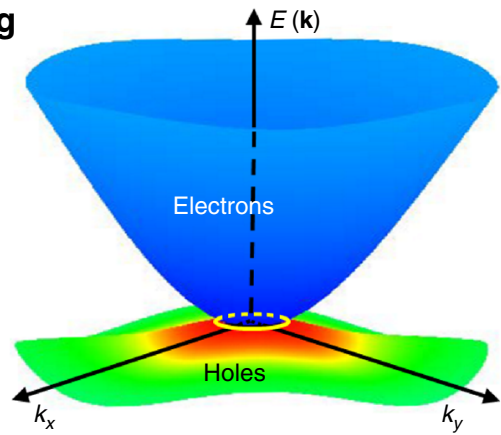

Fig. 1 Excitonic insulator in inverted InAs/GaSb quantum wells tuned by double gates. a Phase diagram for an electron-hole (e-h) system in the parameter space of temperature and e-h pair density. b Pink circle: an e-h plasma (or a semimetal). c Purple diamond: the excitonic insulator, where electrons and holes are weakly bound, like Cooper pairs. $\mathbf{d}$ Red square: an exciton gas consisting of bosonic particles with a finite center-of-mass momentum. e Blue triangle: exciton Bose-Einstein condensate, where the exciton states are degenerate. In $\mathbf{b}$-e, the blue dots represent electrons, the red points are holes, the dashed ellipses indicate the strong binding between electrons and holes, the dashed lines with arrows mean the weak binding between electrons and holes, and the single arrows show the center-of-mass movement of excitons. $\mathbf{f}$ Sketch of a device with front and back gates. The back-gate is used to fix $p_{0}$, and sweeping the front-gate voltage allows us to find a resistance peak at the charge-neutral-point, $n_{0} \sim p_{0}$. $\mathbf{g}$ Band structure of InAs/GaSb quantum wells calculated using the 8-band $\mathbf{k} \cdot \mathbf{p}$ method, for low $n_{0}$ 

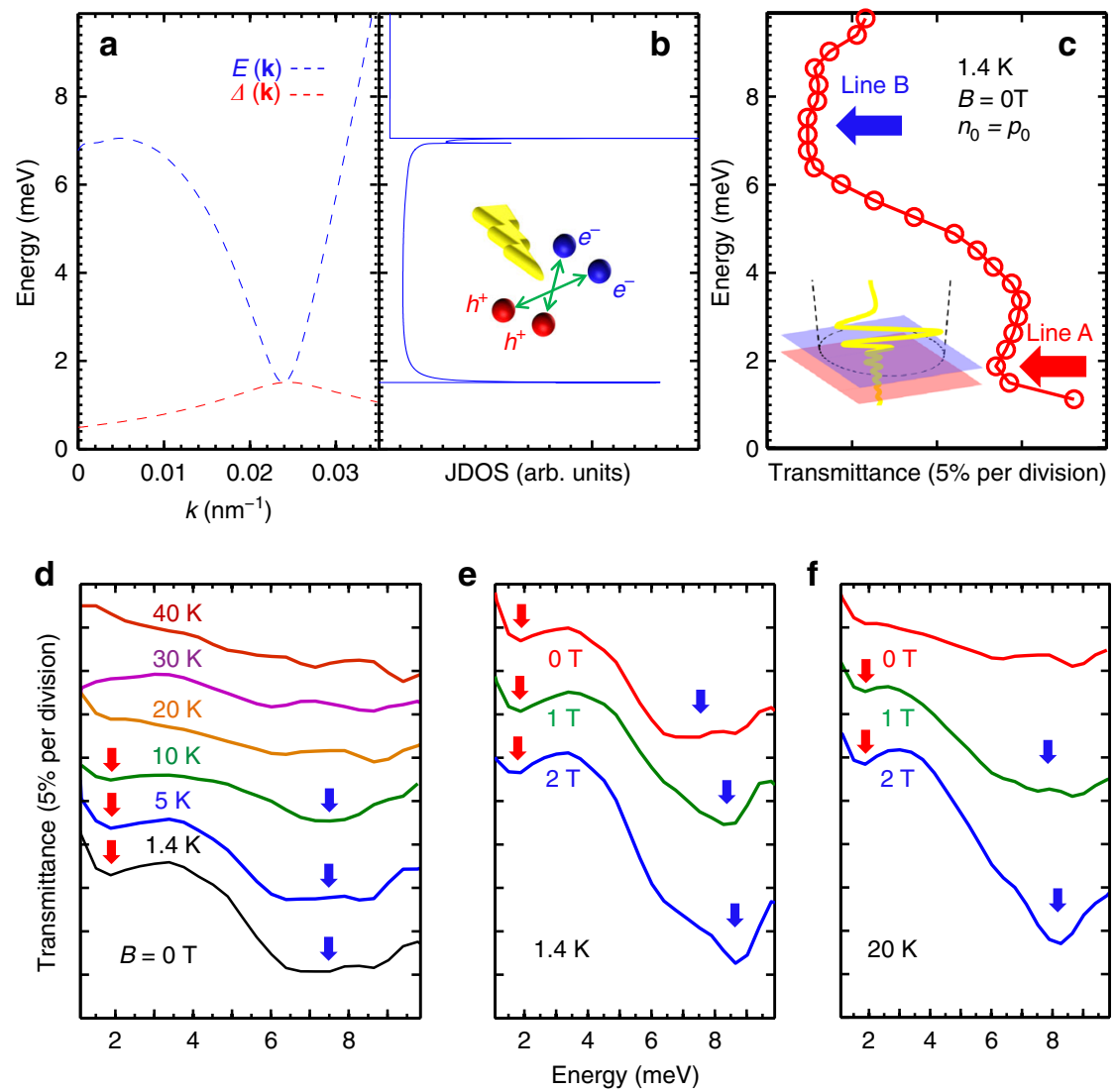

Fig. 2 Pair-breaking excitation spectra of excitonic insulator. a Gap function $\Delta(\mathbf{k})$ (red dashed line) and the pair-breaking energy $E(\mathbf{k})$ (blue dashed line) of the exciton as a function of $k$ for $V_{b}=0 \mathrm{~V}$. b Joint density of states as a function of energy. Inset figure: pair-breaking induced by THz light (Blue dots: electrons. Red dots: holes. Yellow arrow: THz light). c Transmission spectrum at the CNP at $1.4 \mathrm{~K}$ and $0 \mathrm{~T}$ for $V_{\mathrm{b}}=0 \mathrm{~V}$. In the inset figure, the purple layer represents the InAs quantum-well, whereas the red layer represents the GaSb quantum-well. The yellow wave indicates the THz light, and the dashed black circle marks the lateral extent of the focused $\mathrm{THz}$ beam at the sample position. The gate and contacts are not drawn here. $\mathbf{d}$ Transmittance spectra at various temperatures at $0 \mathrm{~T}$. e, f Transmittance spectra at different magnetic fields at 1.4 and $20 \mathrm{~K}$, respectively. The spectra are vertically offset for clarity. The measurement uncertainty in $\mathrm{THz}$ energy is $\pm 0.2 \mathrm{meV}$

electronic transport measurements also suggested the existence of a gap, determining the gap energy to be $\sim 2 \mathrm{meV}$ (or $\sim 25 \mathrm{~K}$ ). We found the gap value to be roughly independent of the strength of an applied in-plane magnetic field but close quickly with increasing $n_{0}$ or temperature. Together with theoretical analysis, we interpret these results as the observation of EI gap opening in this $2 \mathrm{D}$ equilibrium $\mathrm{e}-\mathrm{h}$ gas, suggesting that a $2 \mathrm{D}$ EI phase is realized in our QWs. Moreover, the system exhibited helical edge transport behavior, which supports the notion that the observed low-temperature $\mathrm{e}-\mathrm{h}$ phase is a topological EI.

\section{Results}

Devices. Our devices were made from inverted InAs/GaSb QWs grown by molecular beam epitaxy ${ }^{18,19}$ (also see Methods and Supplementary Figs. 1 and 2). Transport measurements were performed on wafer $\mathrm{A}$ and $\mathrm{C}$, in which a conducting GaAs or $\mathrm{GaSb}$ substrate serves as a back-gate. In this structure (Fig. 1f), the back-gate bias voltage $\left(V_{\mathrm{b}}\right)$ was limited to the negative range, $V_{\mathrm{b}} \leq 0$; starting with $V_{\mathrm{b}}=0$ and with increasing negative bias, we were able to introduce more holes $(p)$ in GaSb QW. Correspondingly, we could sweep the front-gate bias $V_{\mathrm{f}}$ (for electrons in InAs QW) and reach the charge-neutrality point (CNP), i.e., $n_{0}=p_{0}$. The lowest $n_{0}$ in our devices was $\sim 5.5 \times 10^{10}$ $\mathrm{cm}^{-2}$, which was achieved at $V_{\mathrm{b}}=0$. Figure $1 \mathrm{~g}$ shows the band structure of InAs/GaSb QWs calculated using the 8-band k·p method for low $n_{0}$ at $V_{\mathrm{b}}=0$. The average inter-exciton in-plane distance, $2 r_{\text {avg }}$, corresponding to $n_{0} \sim 5.5 \times 10^{10} \mathrm{~cm}^{-2}$, defined through $1=n_{0} \cdot \pi r_{\mathrm{avg}}^{2}$, is $\sim 48 \mathrm{~nm}$. This value should be compared with the effective Bohr radius, $a_{\mathrm{B}}$, which is estimated to be $\sim 30 \mathrm{~nm}$ within a simple effective-mass approximation ${ }^{15}$ using the following parameters ${ }^{22}$ : electron effective mass $m_{\mathrm{e}}^{*} \sim 0.032 m_{0}$ $\left(m_{0}=9.11 \times 10^{-31} \mathrm{~kg}\right)$, hole effective mass $m_{\mathrm{h}}^{*} \sim 0.136 m_{0}$, dielectric constant $\varepsilon \sim 15$, and interlayer distance between the centers of the electron and hole wells $d \sim 10 \mathrm{~nm}$. Therefore, we have $r_{\mathrm{avg}} / a_{\mathrm{B}}$ $\sim 0.8$, indicating strong wavefunction overlap between excitons, a situation reminiscent of Cooper pairs.

Theoretical model. The EI state is expected to have distinctly different optical and transport properties from an exciton BEC. Our system is described by the Hamiltonian ${ }^{3,7-9}$ :

$$
\begin{aligned}
& \hat{h}_{\mathrm{e}-\mathrm{h}}=\sum_{\mathrm{k}}\left(E_{\mathrm{k}}^{\mathrm{e}} \mathrm{a}_{\mathrm{k}}^{\dagger} \mathrm{a}_{\mathrm{k}}+E_{\mathrm{k}}^{\mathrm{h}} b_{\mathrm{k}}^{\dagger} b_{\mathrm{k}}\right) \\
& +\frac{1}{2} \sum_{\mathrm{k}, \mathrm{k}^{\prime}, \mathrm{q}}\left(V_{\mathrm{q}}^{\mathrm{ee}} \mathrm{a}_{\mathrm{k}+\mathrm{q}}^{\dagger} \mathrm{a}_{\mathrm{k}^{\prime}-\mathrm{q}}^{\dagger} \mathrm{a}_{\mathrm{k}^{\prime}} \mathrm{a}_{\mathrm{k}}+V_{\mathrm{q}}^{\mathrm{hh}} b_{\mathrm{k}+\mathrm{q}}^{\dagger} b_{\mathrm{k}^{\prime}-\mathrm{q}}^{\dagger} b_{\mathrm{k}^{\prime}} b_{\mathrm{k}}-2 V_{\mathrm{q}}^{\mathrm{eh}} \mathrm{a}_{\mathrm{k}+\mathrm{q}}^{\dagger} b_{\mathrm{k}^{\prime}-\mathrm{q}}^{\dagger} b_{\mathrm{k}^{\prime}} \mathrm{a}_{\mathrm{k}}\right)
\end{aligned}
$$

where $E_{\mathrm{k}}^{\mathrm{e}, \mathrm{h}}$ are the single-particle electron and hole energies, $\mathbf{k}$ is the in-plane momentum, $a_{\mathrm{k}}^{\dagger}\left(a_{\mathrm{k}}\right)$ and $b_{\mathrm{k}}^{\dagger}\left(b_{\mathrm{k}}\right)$ are the creation (annihilation) operators for electrons in the conduction and holes in the valence bands, respectively, $V_{\mathrm{q}}^{\mathrm{ee}}=V_{\mathrm{q}}^{\mathrm{hh}}=\frac{e^{2}}{2 \varepsilon|\mathrm{q}|}$, and $V_{\mathrm{q}}^{\mathrm{eh}}=$ $V_{\mathrm{q}}^{\mathrm{hh}}=\frac{e^{2}}{2 \varepsilon \mathrm{q}} \mathrm{e}^{-\mathrm{q} \cdot \mathrm{d}}$, where $\varepsilon$ is the dielectric constant. 
a

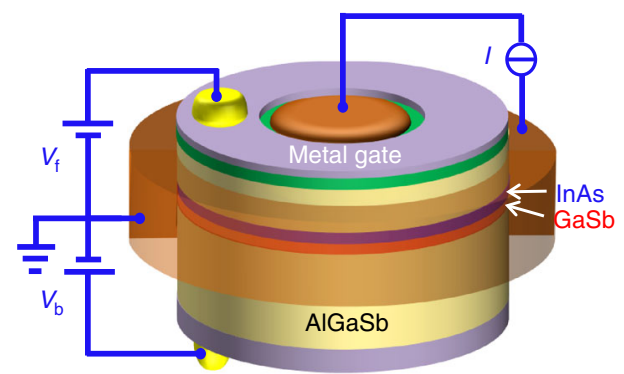

d

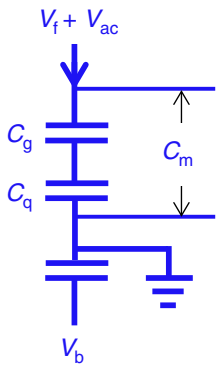

e

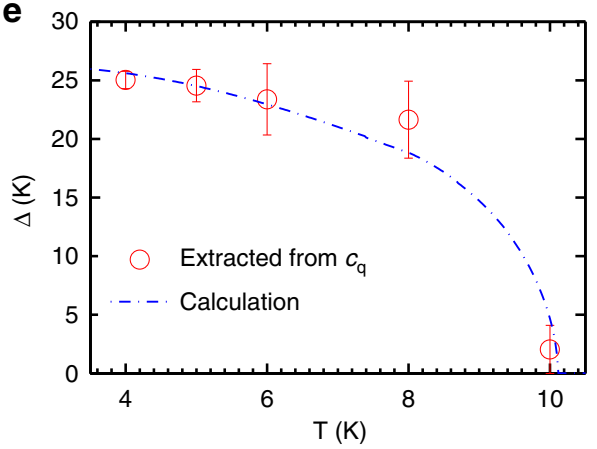

b

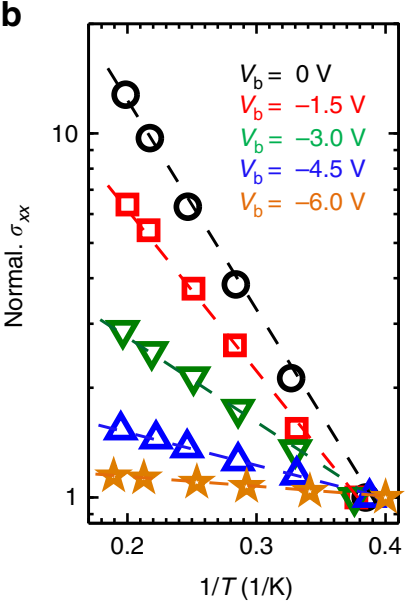

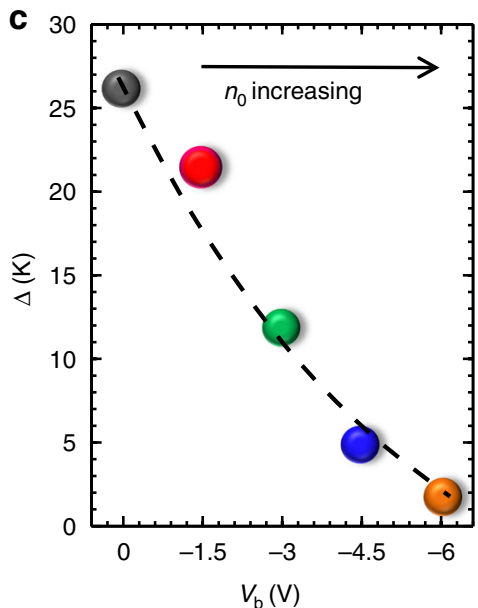

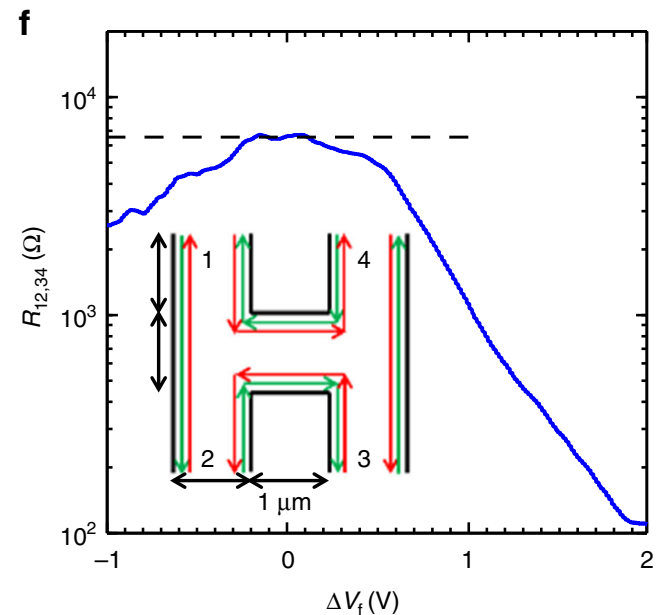

Fig. 3 Excitonic insulator gap and topological edge states. a Sketch of the measurement setup. $\mathbf{b}$ Arrhenius plot of the conductance minimum for different back-gate bias voltages. The data can be fit by $\sigma_{x x} \propto \exp \left(-\Delta / 2 k_{b} T\right)$ to obtain $\Delta$. Here the $\sigma_{x x}$ is normalized by its value at $2.5 \mathrm{~K}$. Dashed lines are guides to the eye. Black circles are for $V_{b}=0 V$, red squares are for $V_{b}=-1.5 \mathrm{~V}$, green down-triangles are for $V_{b}=-3 \mathrm{~V}$, blue up-triangles are for $V_{b}=-4.5 \mathrm{~V}$, and yellow stars are for $V_{b}=-6 \mathrm{~V}$. c Measured gap energy $\Delta$ as a function of back-gate bias voltage. $\mathbf{d}$ Diagram for capacitance measurement. In addition to the dc $V_{f}$ and $V_{b}$, a small low frequency ac voltage was applied to the front-gate, and the capacitance between the front-gate and the quantum wells was measured. e Gap energy as a function of temperature for $V_{b}=0 \mathrm{~V}$. At the charge-neutral point, the gap energies were extracted from $c_{\mathrm{q}}$. The dotteddashed line is from the calculation. The error bars come from the uncertainties in $c_{q}$ and the temperature. $\mathbf{f}$ Nonlocal measurement performed in a mesoscopic $\mathrm{H}$-bar for $\mathrm{V}_{\mathrm{b}}=0 \mathrm{~V}$ at $\mathrm{OT}$ and $30 \mathrm{mK}$. The electrical current is passed through contacts 3 and 4 , and the voltage is measured between contacts 1 and 2. The dashed line indicates the expected resistance value calculated from the Landauer-Büttiker formula. The edge current path is shown in the inset as red and green arrows

To solve this many-body interaction problem, we used a meanfield treatment ${ }^{3,7-9}$ to calculate the exciton dispersion, $E(\mathbf{k})$, and the gap function $\Delta(\mathbf{k})$ (Fig. 2a). Similarly to the case of Cooper pairs, the e-h Coulomb correlations in the EI phase lead to an unstable Fermi surface, spontaneously opening a BCS-like gap (EI gap) $\Delta_{\max }$ near the CNP reached by the Fermi level; this is the gap probed by transport measurements. On the other hand, as a ground state, the EI state absorbs incident photons, with individual electrons and holes in the final state. $E(\mathbf{k})$ is the pair-breaking excitation spectrum: the energy cost of taking one loosely bound exciton out of the condensate and placing a pair of an individual electron and an individual hole in-plane-wave states of momentum $\mathbf{k}$. Figure $2 \mathrm{~b}$ shows the joint density of states (JDOS) we calculated, which predicts two singularity peaks: one peak near $E_{\min } \sim 1.5 \mathrm{meV}$ (or $\sim 18 \mathrm{~K}$ ), and the other near $E(\mathbf{k}) \sim$ $7 \mathrm{meV}$ (or $\sim 80 \mathrm{~K}$ ) with $\mathbf{k} \sim 0$. There is fine structure in the $7 \mathrm{meV}$ peak due to the existence of two nearby peaks. Note that the $E(\mathbf{k})$ and $\Delta(\mathbf{k})$ curves in Fig. 2a are general EI characteristics, independent of material details ${ }^{3,7-9}$, making an EI distinguishable from an exciton BEC (or an exciton gas). For an exciton BEC/ exciton gas, the two peaks merge at $\mathbf{k}=0$, corresponding to the exciton binding energy; furthermore, there is no spontaneous gap opening near the Fermi surface ${ }^{7}$. In the following, from both optics and transport perspectives, we present key findings that support these calculation results, thereby evidencing the existence of an EI in our system.

Pair-breaking excitation measurement of excitonic insulator by terahertz spectroscopy. We performed low-temperature $\mathrm{THz}$ transmission spectroscopy experiments ${ }^{16,23,24}$ (see Methods) on a device covered by a $5 \mathrm{~mm} \times 5 \mathrm{~mm}$ semi-transparent gate, in a frequency (energy) range of $0.25-2.4 \mathrm{THz}(\sim 1-10 \mathrm{meV})$. Note for $\mathrm{THz}$ experiment the wafer $\mathrm{B}$ is used which was prepared on a semi-insulating GaAs substrate. A transmittance spectrum when the system is at the CNP at $1.4 \mathrm{~K}$ is shown in Fig. 2c. Here, two absorption lines (or transmission dips) are present in the spectrum, line $\mathrm{A}$ at $\sim 2 \mathrm{meV}$, and line $\mathrm{B}$ at $7.3 \mathrm{meV}$, consistent with what we expect from Fig. 2a and b. We interpret line A as coming from pair-breaking excitation near the Fermi level and line $\mathrm{B}$ corresponding to excitation near $\mathbf{k}=0$. Mention that what we are measuring is the joint density of states of the pair-breaking excitation process, which is characterized by the spectrum $E(\mathbf{k})$, not a direct extraction of the gap. It should be noted that these features are observed only when $n_{0}$ is low $\left(\sim 5.5 \times 10^{10} \mathrm{~cm}^{-2}\right)$; they disappear in an electron- or hole-dominating regime, or in a high 

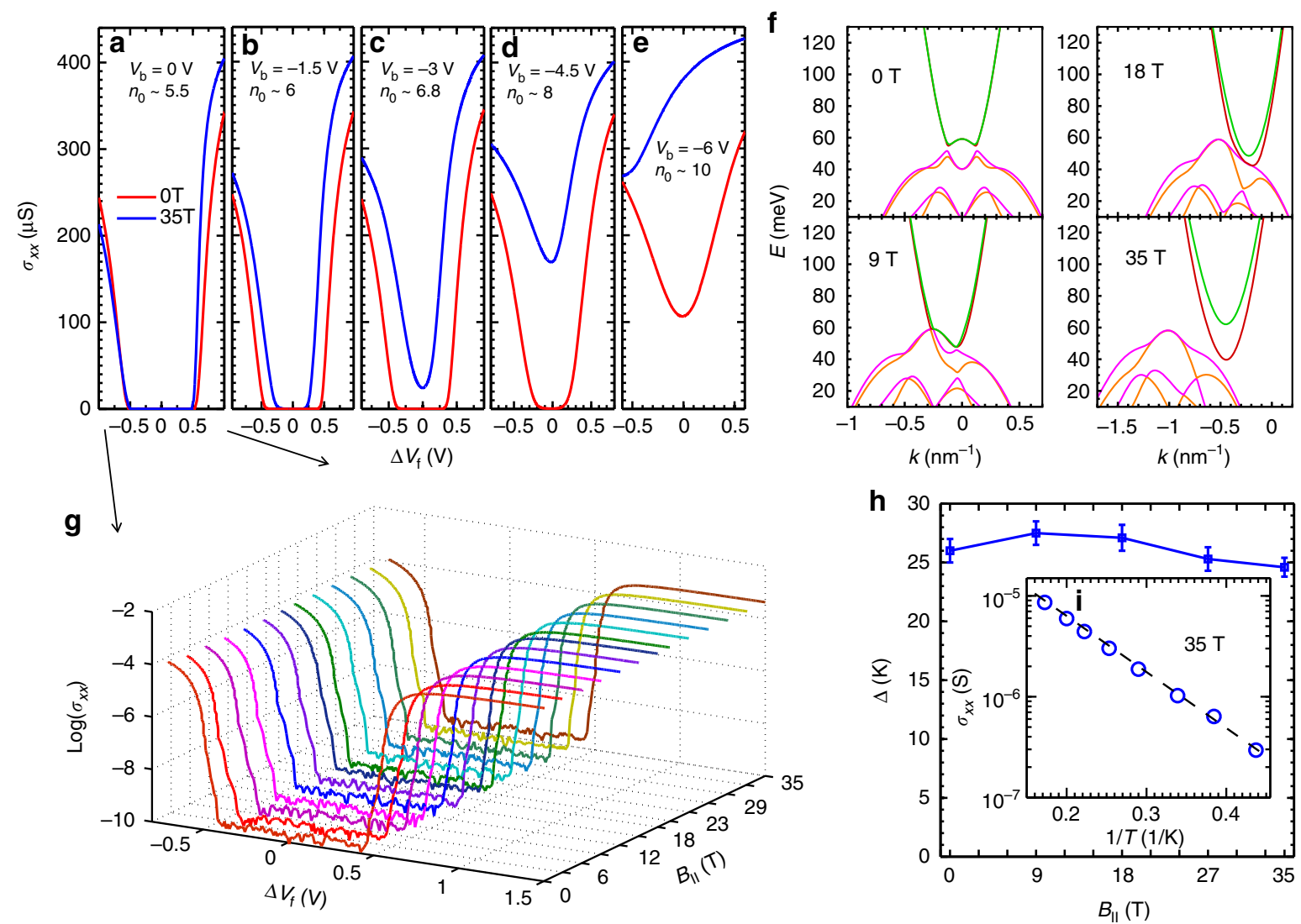

Fig. 4 Measurement of density-dependent hybridization gap under in-plane magnetic fields. a-e Front-gate bias voltage dependence of $\sigma_{x x}$ for a device at different back-gate bias voltages from $0 \mathrm{~V}$ to $-6 \mathrm{~V}$ at $30 \mathrm{mK}$, with a decrement of $1.5 \mathrm{~V}$. In each panel, the value of $n_{0}$ is noted in units of $10^{10} \mathrm{~cm}^{-2}$. The blue (red) lines are for $B_{/ /}=35 \mathrm{~T}(\mathrm{OT})$. f Energy dispersions calculated with an 8-band self-consistant model for tunneling electrons and holes with a typical inverted band at $B_{/ /}=0 \mathrm{~T}, 9 \mathrm{~T}, 18 \mathrm{~T}$, and $35 \mathrm{~T}$. $\mathbf{g}$ Front-gate bias voltage dependence of $\sigma_{x x}$ for a Corbino device at zero back-gate at various $B / /$ from O T to $35 \mathrm{~T}$ measured at $30 \mathrm{mK}$. A zero-conductance dip appears and persists up to $35 \mathrm{~T}$. $\mathbf{h}$ Gap energy $\Delta$ vs. $B / /$ at zero back-gate bias. The error bars come from the uncertainty in extraction of gap energy from Arrhenius plot. i Arrhenius plot of the dip conductance $\sigma_{x x}$ at $35 \mathrm{~T}$ for two orders of magnitude. Dashed line is a guide to the eye

$n_{0}\left(\sim>10^{11} \mathrm{~cm}^{-2}\right)$ case. In the case of line $\mathrm{B}$, in addition to the fine structure in JDOS (Fig. 2b), inhomogeneous broadening due to random potential fluctuations would contribute to the linewidth $^{25}$. Moreover disorder has stronger effect to low $\mathbf{k}$ states that have lower energy, contributing to a broader line. Also, random potential fluctuations are expected to enhance the exciton density at low $\mathbf{k}^{10}$.

The interpretation of the lines as pair-breaking excitations across the BCS-like gap of an EI state is also supported by their temperature dependence (Fig. $2 \mathrm{~d}$ ). At $5 \mathrm{~K}$, the unique two-line structure is still present, which cannot be explained by any singleparticle gap in this system. The absorption amplitude of the lines, which directly reflects the JDOS of the excitations, significantly decreases when the temperature is raised to $10 \mathrm{~K}$. At higher temperatures, the two-line structure is absent. A marked decrease of intensity of both lines occurs at a temperature $(10 \mathrm{~K})$ that is much less than the line energies ( 25 and $90 \mathrm{~K}$, respectively). These results cannot be explained by a single-particle gap but can be interpreted as the transition from an EI state into a metallic state with a critical temperature $T_{\mathrm{c}} \sim 10 \mathrm{~K}$, which also agrees with transport results as shown below.

When a magnetic field perpendicular to the QWs, $B_{\perp}$, is applied at $1.4 \mathrm{~K}$, the amplitudes of the absorption lines increase and line B slightly blue-shifts with the magnetic field, as shown in Fig. 2e. This $B_{\perp}$-induced enhancement of the absorption lines is particularly marked at $20 \mathrm{~K}$, where the lines are initially absent at $0 \mathrm{~T}$, but the $B_{\perp}$ causes them to re-emerge (Fig. 2f). The strengthened absorption under $B_{\perp}$ is likely due to the fact that a $B_{\perp}$ makes e-h pairs more tightly bound ${ }^{7,26}$, hence more stable against dissociation. Furthermore, it has theoretically been predicted that a magnetic field tends to stabilize the EI phase ${ }^{27,28}$, which is in stark contrast to BCS superconductors involving Cooper pairs (which are destroyed by a magnetic field).

Although the two lines (lines A and B) have different energies, they disappear at the same $T_{\mathrm{c}}$ and reappear simultaneously in $B_{\perp}$. Such correlation can be quantitatively understood: both lines are associated with transitions from the EI state to distinct final states with different $\mathbf{k}$ values, as shown in the calculated spectrum (Fig. 2a). It not only confirms that the lines have the same origin but also provides optical spectroscopic evidence for the spontaneous formation of an EI gap through the Coulomb attraction between spatially separated electrons and holes.

Excitonic insulator gap by Corbino measurement. To access the gap function (order parameter) of the EI state, bulk conductance measurements (presented in Figs. 3 and 4) are necessary, and hence, we utilized a Corbino device (Fig. 3a) to exclusively measure bulk properties ${ }^{19}$. The red trace in Fig. 4a shows that the bulk conductivity drops fast with a conductance dip coming to zero at the CNP. Red lines from Fig. $4 \mathrm{a}$ (low $n_{0}$ case, $V_{\mathrm{b}}=0$ ) to Fig. 4e (high $n_{0}$ case, $V_{b}=-6 \mathrm{~V}$ ), the width of the zeroconductance region decreases; the $\sigma_{x x}$ dip around the CNP is lifted from zero, indicating that the EI state is weakened by 
increasing $n_{0}$. The opening of a hard gap in the low $n_{0}$ case can be confirmed quantitatively via thermal activation measurements. The $\sigma_{x x}$ at CNP vs. $1 / T$ can be fit over one order of magnitude in conductivity with an Arrhenius function $\sigma_{x x} \propto \exp \left(-\Delta / 2 k_{\mathrm{b}} T\right)$, with activation energy $\Delta \sim 2 \mathrm{meV}$ (or $\sim 25 \mathrm{~K}$ ) (black dashed line in Fig. 3b, also see Supplementary Fig. 3 and Supplementary Note 1). Our calculated gap energy (blue dashed line in Fig. 2a) agrees with this energy value well.

Next, we measured the gap as a function of $n_{0}$. The data taken at the CNP follows the relation $\sigma_{x x} \propto \exp \left(-\Delta / 2 k_{\mathrm{b}} T\right)$ well, yielding a set of $\Delta$ values as plotted in Fig. 3c. The gap energy diminishes steeply to nearly zero as $n_{0}$ increases (Fig. $3 c$ ). Hence, the gap energy is strongly correlated with $1 / n_{0}$, suggesting that a low $n_{0}$ is crucial for the formation of an EI gap. These results can be understood as a consequence of weakened $\mathrm{e}-\mathrm{h}$ binding through increased screening as the $n_{0}$ increases.

Temperature dependence of excitonic insulator gap by capacitance measurement. On the other hand, as the temperature increases, the EI state becomes unstable and the gap eventually closes. We studied the behavior of the gap as a function of temperature through capacitance spectroscopy experiments (Fig. 3d) (see Methods and Supplementary Fig. 4). $c_{\mathrm{m}}=1 /\left(1 / c_{\mathrm{g}}+\right.$ $\left.1 / c_{\mathrm{q}}\right)$ is the capacitance measured per unit area, where $c_{\mathrm{g}}$ is the geometry capacitance per unit area, $c_{\mathrm{q}}=e^{2} D$ is the quantum capacitance per unit area, and $D$ is the density of states (DOS). We begin with an analysis of the quantum capacitance in different regimes. At $T_{\mathrm{c}} \sim 10 \mathrm{~K}$, the gap vanishes and the $D$ is dominated by electrons and holes; we take $1 / c_{\mathrm{q}}=0$ as a reference point. At $T \sim 0$, the $D$ is zero since it would take an energy cost of $\Delta(T)$ to excite a pair of electron and hole. At an intermediate temperature, $D$ is proportional to $\left(1 / k_{\mathrm{b}} T\right) \exp \left(-\Delta / 2 k_{\mathrm{b}} T\right)$; measurement of $c_{\mathrm{q}}$ is yielding a semi-quantitative estimation of the gap function $\Delta(T)$ as shown in Fig. $3 \mathrm{e}$. We can see that $\Delta$ starts decreasing at $5 \mathrm{~K}$ and finally diminishes at $10 \mathrm{~K}$. This behavior can be well described by the gap function predicted for EI (see Supplementary Fig. 5 and Supplementary Note 2), but is inconsistent with alternative interpretations such as thermal excitations over a single-particle band gap.

Nonlocal measurement of edge states in topological excitonic insulator. In the low $n_{0}$ regime comparable to the case here, the quantization plateau of helical edge states has been previously observed $^{19}$ and taken as the evidence of the QSH effect. Moreover, the plateaus were found to be quite robust under the variance of external parameters such as in-plane magnetic field or temperature. Such a robustness of helical edge states cannot be explained by existing single-particle theory concerning 2D topological insulators. Pikulin and Hyart subsequently proposed $^{20}$ the emergence of an unconventional EI ground state in the inverted InAs/GaSb QWs, providing a plausible explanation for these experimental observations. In their model, the interplay between an excitonic ground state and interlayer tunneling, which is naturally existing in the present InAs/GaSb structure (i.e., without a tunneling barrier between the two layers) can lead to a $p$-wave EI with topologically protected edge states ${ }^{19}$. The present work has provided convincing experimental evidences for the existence of EI gap in this system when it is tuned into low densities.

To explore the nontrivial topological properties of the condensate, we performed nonlocal transport measurements in a mesoscopic H-bar device at low $n_{0}$. According to the Landauer-Büttiker formula (see Supplementary Note 3), for helical edge transport, $R_{12,34}=V_{12} / I_{43}$ should measure quantized resistance of $h / 4 e^{2} \sim 6.45 \mathrm{k} \Omega$. As shown in Fig. $3 \mathrm{f}$, we indeed observed a quantized plateau close to this value, which confirms that helical edge transport ${ }^{29}$ is indeed realized, whereas the bulk is insulating (also see Supplementary Notes 4 and 5, and Supplementary Figs. 6 and 7). Together with the abovepresented optical and transport evidence, these results suggest that an EI spontaneously emerges in the bulk of InAs/GaSb QWs with helical edge modes propagating along the perimeters, consistent with the observed unusual QSH properties in InAs/ $\mathrm{GaSb}^{19}$ within the picture of the proposed topological $\mathrm{EI}^{20}$.

Measurement of density-dependent hybridization gap under in-plane magnetic fields. In InAs/GaSb QWs without a middle barrier layer, conduction band/valence band hybridization is a potential mechanism for the opening of a bulk gap. However, its contribution to the gap can be distinguished by the application of an in-plane magnetic field $B_{/ /}{ }^{30}$. Within the single-particle picture, electrons and holes with the same momentum tunnel between QWs, forming a hybridization gap. An applied $B_{/ /}$ induces a relative shift of band dispersions by the amount $e B_{/ /} d / h$. Consequently, the inter-well tunneling is suppressed due to momentum mismatch, or in other words, $B_{/ /}$creates an effective barrier for tunneling. As shown in our 8-band self-consistent calculations (Fig. 4f) in the tunneling regime (see Supplementary Notes 6 and 7, and Supplementary Fig. 8), as $B_{/ /}$increases beyond $18 \mathrm{~T}$, the two bands will separate in $\mathbf{k}$ space, and the system becomes a semimetal. Here, we applied a $B_{/ /}$of $35 \mathrm{~T}$ to the Corbino device (also see Supplementary Note 8 and Supplementary Fig. 9) and took the conductance increment $\left(\sigma_{x x}(35 \mathrm{~T})-\right.$ $\left.\sigma_{x x}(0 \mathrm{~T})\right)$ at the CNP as a qualitative measure of the contribution from single-particle hybridization to the gap. For $V_{\mathrm{b}}=-6 \mathrm{~V}$ (the highest $\left.n_{0} \sim 1 \times 10^{11} \mathrm{~cm}^{-2}\right), \sigma_{x x}(35 \mathrm{~T})$ increases from the $B=0$ value by four times and the dip vanish at $35 \mathrm{~T}$, indicating that the hybridization effect is dominant. As $n_{0}$ decreases, $\sigma_{x x}(35 \mathrm{~T})$ still deviates from $\sigma_{x x}(0 \mathrm{~T})$ but has a dip at the CNP, showing that the hybridization has a weaker role and the EI gap gradually appears. At $V_{\mathrm{b}}=0 \mathrm{~V}$ (lowest $n_{0} \sim 5.5 \times 10^{10} \mathrm{~cm}^{-2}$ ), $\sigma_{x x}$ is characteristically the same as that at $B=0$, which is further demonstrated by a plot of $\sigma_{x x}$ at various $B_{/ /}$with a broad zero-conductance region seen from $B_{/ /}=0$ to $35 \mathrm{~T}$ (Fig. $4 \mathrm{~g}$ ). In the presence of a $B_{/ /}, \sigma_{\mathrm{xx}}$ markedly depends on $n_{0}$, demonstrating that the EI phase is more stable at a lower $n_{0}$. At the lowest $n_{0}$, the gap remains open in spite of strong in-plane magnetic fields, confirmed by activation measurements under different $B_{/ /}$up to $35 \mathrm{~T}$ in Fig. $4 \mathrm{~h}$ and $\mathrm{i}$. The observed systematic responses of the gap conductance to the $B_{/ /}$ support the notion that the EI phase, not the hybridization effect, is responsible for the appearance of the gap in such a low density, equilibrium electron-hole gas.

\section{Discussion}

The realization of an excitonic insulator state in our InAs/GaSb system paves the way for further studying many-exciton physics in great detail as well as depth (e.g., BCS-BEC crossover, and BEC exciton) in an equilibrium electron-hole system without optical pumping. Due to the weak binding nature of the EI and a lack of middle barrier, counter-flow experiments on the current system would not be as effective as in the BEC case $\mathrm{C}^{12}$. With an additional $\mathrm{AlGaSb}$ barrier between the electron and hole layers, counter-flow studies would be enabled to explore BCS to BEC crossover. Moreover, the inverted band structure of the current system brings in topological nature to the EI state, which will allow one to study $2 \mathrm{D}$ interacting topological insulators in a highly controllable manner. For example, with a thin AlGaSb middle barrier or utilizing strained-layer InAs/GaInSb QWs, the symmetry of the order parameter can be controlled by tuning the interplay of interlayer interactions and tunneling. 


\section{Methods}

Characterization and transport measurements. $\mathrm{Al}_{0.8} \mathrm{Ga}_{0.2} \mathrm{Sb}-\mathrm{InAs} /$

$\mathrm{GaSb}-\mathrm{Al}_{0.8} \mathrm{Ga}_{0.2} \mathrm{Sb}$ wafers were prepared by molecular beam epitaxy (MBE) on (001) substrate with a $1 \mu \mathrm{m}$ thick buffer layer, with the following nominal parameters: Wafer A, on $\mathrm{N}+$ GaAs substrate, with $12.5 \mathrm{~nm}$ InAs/10 nm GaSb QWs; Wafer B, on SI- GaAs substrate, with $11.5 \mathrm{~nm}$ InAs/8 nm GaSb QWs; Wafer C, on $n$-GaSb substrate, with $11 \mathrm{~nm}$ InAs/7 nm GaSb QWs. In Wafer A and B, the interface between the GaSb and InAs QWs was doped with a dilute sheet of Si with a concentration of $\sim 1 \times 10^{11} \mathrm{~cm}^{-2}$, whereas there was no doping in Wafer C.

Characterization of wafer A can be found in ref. ${ }^{19}$. In wafer A, Supplementary Fig. $2 \mathrm{a}$ and b shows $B / e R_{x y}$ vs. $\Delta V_{\mathrm{f}}$ for a Hall bar device at $V_{\mathrm{b}}=-6 \mathrm{~V}$ and $0 \mathrm{~V}$, respectively; $B$ is the perpendicular magnetic field and $\Delta V_{\mathrm{f}}$ is the front-gate bias increment from the CNP. For $V_{\mathrm{b}}=-6 \mathrm{~V}$, at the high electron density (regime I), $B$ / $e R_{x y}$ is consistent with the density obtained from Subinikov de Haas oscillations, linear with $V_{\mathrm{f}}$. As the top of the hole band is reached by the Fermi level, holes are introduced and two-carrier transport dominates, with $R_{x y}$ traces divergent and the system reaching the $\mathrm{e}-\mathrm{h}$ hybridized regime (regime II). Owing to the absence of a hard gap, $B / e R_{x y}$ is still dominated by electron-hole residual carriers even in the hybridization gap (regime III). As the Fermi level is below the bottom of the electron band, holes dominate and $R_{x y}$ becomes linear again (regime IV). For $V_{\mathrm{b}}=$ $0 \mathrm{~V}$, where less holes are introduced by $V_{\mathrm{b}}$, as the CNP is approached, similarly the system goes from regime i to regime II. However, within regime II, a plateau-like feature is observed, indicating the formation of an EI gap (regime V).

In Wafer $\mathrm{C}$, lattice matched epitaxial layers were grown on a GaSb substrate, which should result in enhanced carrier mobility. The carrier mobility of Wafer C measured at $300 \mathrm{mK}$ indeed showed significant improvement. The typical electron mobility was $90,000 \mathrm{~cm}^{2} \mathrm{~V}^{-1} \mathrm{~s}^{-1}$ for a density of $5 \times 10^{11} \mathrm{~cm}^{-2}$, and the hole mobility was about one order of magnitude lower at similar densities. Magnetotransport experiments showed well-resolved quantum oscillations, as shown in Supplementary Fig. 1a. In a perpendicular magnetic field, the device can be tuned by the front-gate from the electron dominating regime to the hole-dominating regime in an asymmetrical Hall bar, as shown in Supplementary Fig. 1b. As the electron density decreases, holes emerge, which bends the trace. When the hole and electron densities become comparable, the trace drops again with an EI gap emerging and helical edges dominating. The equilibrium density $n_{\mathrm{o}}$ was $\sim 5 \times 10^{10}$ $\mathrm{cm}^{-2}$.

The $V_{\mathrm{f}}$ dependence of the conductance in a Corbino device $\mathrm{C} 2$ (made from Wafer C) is shown in Supplementary Fig. 1c. Similar to Wafer A, there is a conductance dip at the CNP with the resistivity equal to $\sim 1 \mathrm{M} \Omega /$ square. As we apply $B_{/ /}$up to $35 \mathrm{~T}$, the dip conductance stays nearly the same, which confirms that this gap is not from hybridization, as shown in Supplementary Fig. 1d. Furthermore, the constant dip conductance also shows that the gap does not close between $0 \mathrm{~T}$ and $35 \mathrm{~T}$. These findings are very similar to those from Wafer A, suggesting that $\mathrm{Si}$ doping is not essential for the emergence of the EI gap.

Terahertz measurements. In optical spectroscopy experiments, we measured the transmission of a terahertz $(\mathrm{THz})$ beam through the device made from Wafer $\mathrm{B}$, which was prepared on a semi-insulating GaAs substrate. The device had a semitransparent front-gate, and the gated area was $5 \mathrm{~mm} \times 5 \mathrm{~mm}$ for maximizing the $\mathrm{THz}$ transmission. Through the semi-transparent front-gate, we modulated the system between the CNP and the low mobility electron regime. The electron regime we selected had low mobility and low density, so it had no feature in the $0.25-2.4 \mathrm{THz}$ frequency range, working as a good reference for the exciton-induced transmission. The transmittance was defined as the intensity of the $\mathrm{THz}$ beam transmitted through the sample normalized by the reference signal.

\section{Capacitance-voltage measurements. We performed capacitance-voltage (CV)} measurements ${ }^{30}$ under different $V_{\mathrm{b}}$ in Corbino devices $\mathrm{C} 1$ (Wafer A) and C2 (Wafer C) (Supplementary Fig. 4a). In addition to the dc $V_{\mathrm{f}}$ and $V_{\mathrm{b}}$, a small ac $(f=$ $100 \mathrm{~Hz}$ ) voltage was applied to the front-gate, and the capacitance between the front-gate and the QWs was measured. In CV measurements ${ }^{30}$, the geometry capacitance $c_{\mathrm{q}}$ and quantum capacitance $c_{\mathrm{q}}=e^{2} D$ contribute to the measured capacitance per unit area $c_{\mathrm{m}}$ as $c_{\mathrm{m}}=1 /\left(1 / c_{\mathrm{g}}+1 / c_{\mathrm{q}}\right)$, where $D$ is the density of states (DOS). In the electron or hole-dominating regime, $c_{\mathrm{g}}$ is much smaller than the quantum capacitance, so $c_{\mathrm{g}}$ dominates in $c_{\mathrm{m}}$. When the DOS decreases and quantum capacitance is smaller than $c_{\mathrm{g}}, c_{\mathrm{m}}$ drops and the quantum capacitance dominates.

The out-of-phase signal was one order of magnitude less than the in-phase signal. In this case, for devices from both Wafers $A$ and $C$, the observed capacitance drop near the CNP represented the reduced DOS. For $V_{\mathrm{b}}=-6 \mathrm{~V}$, as illustrated in the blue trace of Supplementary Fig. $4 \mathrm{a}$, the capacitance near the CNP was nearly constant, agreeing with previous $\mathrm{CV}$ results ${ }^{30}$ about the hybridized gap (the tunneling is too weak to form a hard gap). The DOS in this gap can be treated as the mixture of electron's and hole's. For $V_{\mathrm{b}}=0 \mathrm{~V}$, the capacitance at the CNP drops to $10 \%$ of $c_{\mathrm{g}}$, which indicates that the hard gap (EI gap) forms with a reduced DOS. $\mathrm{CV}$ measurements performed on Corbino device C2 with $V_{\mathrm{b}}=0 \mathrm{~V}$ also showed that there is a capacitance drop near the CNP (Supplementary Fig. 4b), confirming that the EI gap exists and the gap is not from Si doping. It should be mentioned that in Wafer $C$ there was no doping and thus, Anderson localization (AL) was suppressed. Moreover, AL could not reduce the DOS. Therefore, the CV results confirm that the hard gap does not originate from AL. A large capacitance drop shows that the co-existing electrons and holes can form neutral particles (reduced DOS) with the formation of the EI gap.

Next we increased the temperature. Supplementary Fig. 4c shows CV traces at $V_{\mathrm{b}}=0 \mathrm{~V}$ for different temperatures. We find that the EI gap starts collapsing at $6 \mathrm{~K}$ and disappears at $10 \mathrm{~K}$. This confirms that the EI gap dominates in the lowtemperature regime, and also provides the temperature window for thermal activation energy measurements.

Determination of equilibrium density of electron and hole. Ideally, the electrons are tuned by the front-gate, whereas the holes are tuned by the back-gate. Owing to the imperfect screening from the electron layer, the front-gate could simultaneously modulate the electrons and holes when holes appear.

Supplementary Fig. 12a shows that, for $\Delta V_{\mathrm{f}}$ sweeping from $2 \mathrm{~V}$, the electron density is high and linear with $\Delta V_{\mathrm{f}}$, so we can extract the electron density increment per $\Delta V_{\mathrm{f}}$. Supplementary Fig. 12b shows the electron density rate under different $V_{\mathrm{b}}$. The rate is constant for $\left|V_{\mathrm{b}}\right|<3 \mathrm{~V}$. When $\left|V_{\mathrm{b}}\right|$ increases and holes appear, the rate decreases until $V_{\mathrm{b}}=-7.5 \mathrm{~V}$, where the rate saturates.

From CV measurements, we know that the capacitance stays constant until the EI gap appears. The capacitance per unit area $c_{\mathrm{m}}$ means the absolute density $(|n|+\mid$ pl) increment per $\Delta V_{\mathrm{f}}$. So we can integrate the capacitance over $\Delta V_{\mathrm{f}}$ to obtain the absolute density, as shown in Supplementary Fig. 12a. It should be mentioned that, for the high electron density, the hole density can be neglected, so the absolute density agrees with the electron density. The absolute density decreases with a constant rate until it gets to nearly zero, as shown in the blue dashed line, where the $\mathrm{CNP}$ is approached. In this case, we can obtain the voltage where the CNP is reached (vertical dashed black line).

In the magneto-transport trace (red dotted line in Supplementary Fig. 12a), as the holes emerge, the trace bends up and it is not straight forward to extract the electron density. However it is reasonable to make an approximation that, when holes appear, the electrons change in the saturated rate (green dashed line) until the $\mathrm{CNP}$ is reached, as illustrated in Supplementary Fig. 12a. This way we can estimate the equilibrium density $n_{0}$ (marked by the circle). The density uncertainty is $5 \times$ $10^{9} \mathrm{~cm}^{-2}$. Similarly, we obtain $n_{0}$ under different $V_{\mathrm{b}}$, as shown in Supplementary Fig. 13.

Data availability. The authors declare that the data supporting the findings of this study are available within the paper and its Supplementation Information files or from the corresponding author upon reasonable request.

Received: 27 May 2017 Accepted: 30 October 2017

Published online: 07 December 2017

\section{References}

1. Mott, N. F. The transition to the metallic state. Phil. Mag. 6, 287-309 (1961).

2. Knox, R. S. Theory of excitons. Solid State Phys. Suppl. 5, 100 (1963).

3. Keldysh, L. V. K. \& Kopaev, Y. V. Possible instability of the semimetallic state toward coulomb interaction. Fiz. Tverd. Tela 6, 2791 (1964).

4. Jérome, D., Rice, T. M. \& Kohn, W. Excitonic insulator. Phys. Rev. 158 462-475 (1967)

5. Halperin, B. I. \& Rice, T. M. Possible anomalies at a semimetal-semiconductor transition. Rev. Mod. Phys. 40, 755-766 (1968).

6. Lozovik, Yu. E. \& Yudson, Y. I. Feasibility of superfluidity of paired spatially separated electrons and holes; a new superconductivity mechanism. JETP Lett. 22, 274 (1975).

7. Littlewood, P. B. \& Zhu, X. Possibilities for exciton condensation in semiconductor quantum-well structures. Phys. Scripta T68, 56-67 (1996).

8. Zhu, X., Littlewood, P. B., Hybertsen, M. S. \& Rice, T. M. Exciton condensate in semiconductor quantum well structures. Phys. Rev. Lett. 74, 1633-1636 (1995).

9. Naveh, Y. \& Laikhtman, B. Excitonic instability and electric-field-induced phase transition towards a two-dimensional exciton condensate. Phys. Rev. Lett. 77, 900-903 (1996).

10. Butov, L. V., Gossard, A. C. \& Chemla, D. S. Towards Bose-Einstein condensation of excitons in potential traps. Nature 414, 47-52 (2002).

11. Snoke, D. Spontaneous Bose coherence of excitons and polaritons. Science 298, 1368-1372 (2002).

12. Eisenstein, J. P. \& MacDonald, A. H. Bose-Einstein condensation of excitons in bilayer electron systems. Nature 432, 691-694 (2004).

13. Luin, S. et al. Observation of soft magnetorotons in bilayer quantum Hall ferromagnets. Phys. Rev. Lett. 90, 236802 (2003).

14. Datta, S., Melloch, M. R. \& Gunshor, R. L. Possibility of an excitonic ground state in quantum wells. Phys. Rev. B 32, 2607-2609 (1985).

15. Xia, X., Chen, X. M. \& Quinn, J. J. Magnetoexcitonsin a GaSb-AlSb-InAs quantum-well structure. Phys. Rev. B 46, 7212-7215 (1992) 
16. Kono, J. et al. Far-infrared magneto-optical study of two-dimensional electrons and holes in InAs/AlGaSb quantum wells. Phys. Rev. B 55, 1617-1636 (1997).

17. Liu, C. X., Hughes, T. L., Qi, X. L., Wang, K. \& Zhang, S. C. Quantum spin Hall effect in inverted type-II semiconductors. Phys. Rev. Lett. 100, 236601 (2008).

18. Knez, I., Du, R. R. \& Sullivan, G. Evidence for helical edge modes in inverted InAs/GaSb quantum wells. Phys. Rev. Lett. 107, 136603 (2011).

19. Du, L. J., Knez, I., Sullivan, G. \& Du, R. R. Robust helical edge transport in gated InAs/GaSb bilayers. Phys. Rev. Lett. 114, 096802 (2015).

20. Pikulin, D. I. \& Hyart, T. Interplay of exciton condensation and the quantum spin Hall effect in InAs/GaSb bilayers. Phys. Rev. Lett. 112, 176403 (2014).

21. Seradjeh, B., Moore, J. E. \& Franz, M. Exciton condensation and charge fractionalization in a topological insulator film. Phys. Rev. Lett. 103, 066402 (2009).

22. Mu, X., Sullivan, G. \& Du, R. R. Effective g-factors of carriers in inverted InAs/ GaSb bilayers. Appl. Phys. Lett. 108, 012101 (2016).

23. Wang, X., Belyanin, A. A., Crooker, S. A., Mittleman, D. M. \& Kono, J. Interference-induced terahertz transparency in a semiconductor magnetoplasma. Nat. Phys. 6, 126-130 (2010).

24. Zhang, Q. et al. Collective non-perturbative coupling of $2 \mathrm{D}$ electrons with highquality-factor terahertz cavity photons. Nat. Phys. 12, 1005-1011 (2016).

25. Castella, H. \& Wilkins, J. W. Splitting of the excitonic peak in quantum wells with interfacial roughness. Phys. Rev. B 58, 16186-16193 (1998).

26. Zhang, Q. et al. Stability of high-density two-dimensional excitons against a Mott transition in high magnetic fields probed by coherent terahertz spectroscopy. Phys. Rev. Lett. 117, 207402 (2016).

27. Fenton, E. W. Excitonic insulator in a magnetic field. Phys. Rev. 170, 816-821 (1968).

28. Kuramoto, Y. \& Horie, C. Two-dimensional excitonic phase in strong magnetic fields. Solid State Commun. 25, 713-716 (1978).

29. Roth, A. et al. Nonlocal transport in the quantum spin Hall state. Science 325, 294-297 (2009)

30. Yang, M. J., Yang, C. H., Bennett, B. R. \& Shanabrook, B. V. Evidence of a hybridization gap in "semimetallic" InAs/GaSb systems. Phys. Rev. Lett. 78, 4613-4616 (1997).

\section{Acknowledgements}

We acknowledge helpful conversations with A.H. MacDonald, B.I. Halperin, and D.I. Pikulin. Work at Rice University was supported by NSF Grants No. DMR-1207562 and No. DMR-1508644 (R.-R.D.), and by DOE Grant No.DE-FG02-06ER46274 (L.D.). W.L. and K.C. were supported by NSFC (No. 11434010). X.L. and J.K. acknowledge support from NSF (Grant No. DMR-1310138). A portion of this work was performed at the National High Magnetic Field Laboratory, which is supported by NSF Cooperative Agreement under No. DMR-1157490, and by the State of Florida.

\section{Author contributions}

L.D. fabricated the devices and performed transport experiments. L.D. and X.L. performed the THz experiments under the supervision of J.K., L.D., X.L., J.K., and R.-R.D analyzed the data. W.L. and K.C. developed the theoretical model and performed the numerical calculations. G.S. prepared the MBE semiconductor materials. L.D., J.K., and R.-R.D. co-wrote the manuscript with input from the other authors. L. D. and R.-R.D. conceived the project. R.-R.D. provided overall supervision and coordination of the project.

\section{Additional information}

Supplementary Information accompanies this paper at https://doi.org/10.1038/s41467017-01988-1.

Competing interests: The authors declare no competing financial interests.

Reprints and permission information is available online at http://npg.nature.com/ reprintsandpermissions/

Publisher's note: Springer Nature remains neutral with regard to jurisdictional claims in published maps and institutional affiliations.

(c) Open Access This article is licensed under a Creative Commons Attribution 4.0 International License, which permits use, sharing, adaptation, distribution and reproduction in any medium or format, as long as you give appropriate credit to the original author(s) and the source, provide a link to the Creative Commons license, and indicate if changes were made. The images or other third party material in this article are included in the article's Creative Commons license, unless indicated otherwise in a credit line to the material. If material is not included in the article's Creative Commons license and your intended use is not permitted by statutory regulation or exceeds the permitted use, you will need to obtain permission directly from the copyright holder. To view a copy of this license, visit http://creativecommons.org/ licenses/by/4.0/.

(C) The Author(s) 2017 